\title{
FÖDERALISMUS UNTER DEN BEDINGUNGEN EINES MILITÄRREGIMES: ARGENTINIEN*
}

\author{
Von Peter Malanczuk
}

Die argentinische Republik wird seit dem Militärputsch im März 1976 gegen die rechtsperonistische Regierung unter Maria Estela Martinez de Perón wie in der meisten Zeit der Geschichte seit dem ersten erfolgreichen Staatsstreich im Jahre 1930 wieder von einem konservativen Militärregime regiert. Neben Mexiko, Venezuela und Brasilien gehört Argentinien zu den vier Staaten im lateinamerikanischen Raum, deren Verfassungen eine föderative Gliederung vorsehen. In all diesen Staaten ist jedoch der Föderalismus mehr Fiktion als Wirklichkeit. Die Verfassungstexte in Lateinamerika, die meist nach den Vorbildern der liberalen Verfassungen Frankreichs, der Spaniens von 1812 und vor allen der USA geschaffen wurden, zeugen von einer Geschichte der fehlgeschlagenen Rezeptionen. Sie sind ein Produkt der Uberfremdung und haben unter den ganz anderen historischen, wirtschaftlichen und politischen Gegebenheiten in Lateinamerika kaum mehr als literarische Bedeutung erlangt ${ }^{1}$.

Für den Abstieg des Föderalismus in Argentinien gibt es mannigfaltige wirtschaftliche, politische, technische und verfassungsrechtliche Gründe ${ }^{2}$. Dieser Bericht interessiert sich in erster Linie für die verfassungsrechtlichen Aspekte, obgleich diese sich nicht von den anderen isolieren lassen. Da eine bloße Analyse und Beschreibung des Normenbestandes irreführend ist, muß besonders die Realfunktion des Verfassungsrechtes in dem einst als Vorbild einer liberal-demokratischen Entwicklung gefeierten Land und die Verfassungspraxis in Rechnung gestellt werden, die das bundesstaatliche Prinzip zunehmend ausgehöhlt hat. Von besonderem Interesse ist in diesem Zusammenhang die Frage, in welchem Maße die Herrschaft des Militärs, das sich seit 1930 zum entscheidenden außerkonstitutionellen Machtfaktor in Argentinien entwickelt hat, für das Absinken der Provinzen zu faktisch bloßen Verwaltungseinheiten verantwortlich ist. Die gegenwärtige politische Situation und die Aussichten des argentinischen Föderalismus können mit der Frage nach der Durchführbarkeit einer demokratischen Regierungsform nach nordamerikanischem Muster nur auf dem Hintergrund der allgemeinen Krise der argentinischen Gesellschaft, den sozio-ökonomischen Strukturproblemen und den Ursachen des Phänomens der argentinischen Militärregime beurteilt werden.

Die vergleichende Föderalismusforschung und die Analyse der diversen Erscheinungsformen des bundesstaatlichen Prinzips in der Welt finden in letzter Zeit zunehmende Aufmerksamkeit $^{3}$. Aus methodischen Gründen wegen der unterschiedlichen Gegegebenheiten, der politischen Kultur und der Realfunktion des Verfassungsrechtes ist ein Vergleich der bundesstaatlichen Organisationsformen und Kooperationsweisen in der Verfassungswirklichkeit westlicher Industriestaaten ${ }^{4}$, etwa in den USA, in Kanada, Australien, der Schweiz oder

\footnotetext{
* Dem Aufsatz liegt ein Bericht für die Jahrestagung 1978 des Arbeitskreises für Ubberseeische Verfassungsvergleichung zugrunde, der für die Veröffentlichung überarbeitet und ergänzt wurde. Für ihre hilfreichen Anregungen bin ich Bettina Wieser und Brun-Otto Bryde besonders verpflichtet.

1 Vgl. T. T. Evers, Miltärregierung in Argentinien, Hamburg 1972, S. 21 ff.

2 Vgl. R. H. Dolkart, The Provinces, in: M. Falcoff/R. H. Dolkart (Hrg.), Prologue to Perón, University of California Press 1975, S. $164 \mathrm{ff}$.

3 F. Esterbauer/G. Héraud/P. Pernthaler (Hrg.), Föderalismus als Mittel permanenter Konfliktregelung, Schriftenreihe des Instituts für Föderalismusforschung Bd. 6, Wien 1977; zur CSSR und zu Jugoslawien vgl. die Aufsätze von Kipke und Kulić in 25 JOR NF 1976.

4 Hierzu: M. Bothe, Die Kompetenzstruktur des modernen Bundesstaates in rechtsvergleichender Sicht, Berlin 1977.
} 
der Bundesrepublik Deutschland, mit den Verhältnissen in Argentinien ebensowenig sinnvoll wie mit den wiederum anders gelagerten föderalen Formen, die manche Verfassungen sozialistischer Staaten vorsehen. Dieser Bericht beschränkt sich deshalb auf die Untersuchung des Föderalismus in Argentinien, dessen historische und ökonomische Besonderheiten $^{5}$ auch vor einer vorschnellen Verallgemeinerung im Hinblick auf die Problematik in den anderen lateinamerikanischen Bundesstaaten warnen.

Die Darstellung ist folgendermaßen gegliedert: Zunächst werden die sozio-ökonomischen Strukturprobleme Argentiniens kurz skizziert, auf deren Hintergrund die seit 1928 anhaltende politisch-institutionelle Krise und das Phänomen der argentinischen Militärregime zu verstehen sind (I.). Dann werden der historische Hintergrund des argentinischen Föderalismus im 19. Jahrhundert und die sich wandelnde Bedeutung des „Provinzproblems“ beschrieben (II.). Schließlich sollen dann die Grundzüge der verfassungsrechtlichen Ausgestaltung des Bundesstaatsprinzips und die Verfassungspraxis erörtert werden (III.).

\section{Die Krise Argentiniens und die gegenwärtige politische Situation}

Argentinien galt einst als das Vorbild einer liberal-demokratischen Entwicklung6. Die Jahre zwischen 1880 und 1930 nach der Epoche der Bürgerkriege und inneren Wirren brachten einen für Lateinamerika beispiellosen wirtschaftlichen Aufschwung und eine Phase der scheinbaren politischen institutionellen Stabilität. Nach der Weltwirtschaftskrise entwikkelte sich das Militär zum entscheidenden politischen Machtfaktor in Argentinien und setzten sich zunehmend Gewalt, Manipulation und Korruption als hauptsächliche Mittel der politischen Auseinandersetzung durch. Die argentinische Gesellschaft ist seit dem Aufstieg des Peronismus (1943-1955) durch tiefe und scheinbar unüberbrückbare soziale, politische und ideologische Gegensätze gespalten, die sich in der Konfrontation zwischen dem Militär und den peronistischen Gewerkschaften oder den verschiedenen Guerilla- und Terrorgruppen seit dem Sturz Peróns 1955 zuspitzen, aber nicht vollständig zeigen ${ }^{7}$. Die Spaltung der heterogenen peronistischen Bewegung nach Peróns Tod 1974 in einen rechten und einen linken Flügel hat die Brutalisierung des politischen Prozesses vergrößert, kaum aber die Chancen erhöht, die verkrusteten sozialökonomischen Strukturen der argentinischen Gesellschaft zu verändern und die wirtschaftlichen Probleme des Landes zu lösen. Die inneren Schwierigkeiten sind so gravierend, daß die früheren Träume von einer Führungsrolle Argentiniens in Südamerika oder gar auf der internationalen Ebene ebenso zur Illusion geworden sind wie die Möglichkeiten, eine liberale Demokratie und politische Spielregeln nach angelsächsischem Muster durchzusetzen.

Die politische Kultur des von europäischen Einwanderern, vornehmlich aus Italien und Spanien, besiedelten Landes, die vorherrschenden Ideologien des „Liberalismus“ und des „Nationalismus“, Begriffe wie „Faschismus“ und „Kommunismus“, politische Parteien und Gewerkschaften lassen sich nicht mit europäischen Maßstäben messen und Erscheinun-

\footnotetext{
5 P. Waldmann, Argentinien, in: D. Nohlen/F. Nuscheler (Hrg.), Handbuch der Dritten Welt Bd. 3, Hamburg 1976, S. 13 ff.

6 M. Falcoff/R. H. Dolkart, a. a. O., S. IX; R. Levene, A History of Argentinien, translated and edited by W. S. Robertson, New York 1963.

7 Aus der Literatur: J. Revers, Le péronisme, Rev. Défense Nationale 1972, S. 1861 ff.; J. Deiner, Eva Perón and the Roots of Political Instability in Argentina, Civilisations 1973/74, S. 195 ff.; F. C. Turner, Das Erbe des Peronismus, Europa-Archiv 1977, S. 467 ff.; K. H. Fuchs/P. Hiedl/C. Streubel, Zur neueren Entwicklung in Argentinien, Bl. f. deut. u. int. Pol. 17 (1972), S. 1064 ff.; L. Bieber, Zur Entwicklung in Argentinien unter den peronistischen Regierungen seit 1973, Bl. f. deut. u. int. Pol. 20 (1975), S. 777 ff.; S. Fronius, Nicht besiegt und noch nicht Sieger - Argentinien und die Entwicklung der peronistischen Arbeiterbewegung, Berlin 1977.
} 
gen des gleichen Namens vergleichen ${ }^{8}$. Die neuere historische, sozialwissenschaftliche Forschung stellt diese Besonderheiten in Rechnung, interessiert sich aber in erster Linie für die sozialökonomischen Wurzeln der Dauerkrise des zweitgrößten, modernisierten Landes in Südamerika. Dabei erfahren der Zeitraum um die Jahrhundertwende und die in der argentinischen Geschichtsschreibung als unrühmliche Dekade bezeichnete Zeit zwischen 1930 und 1943 zunehmende Aufmerksamkeit ${ }^{9}$. Die Interpretationen unterscheiden sich je nach dem theoretischen Ansatz und dem herangezogenen empirischen Material. Jedoch lassen sich die Grundprobleme Argentiniens mit dem französischen Historiker und Politikwissenschaftler Alain Rouquié10 als die einer ,,blockierten Gesellschaft" (société bloquée) beschreiben, in der eine beachtliche Kluft zwischen der sozialen Struktur und der gesellschaftlichen Entwicklung seit der Herausbildung einer starken Mittelschicht und der Arbeiterschaft mit der Industrialisierung $\mathrm{zu}$ beobachten ist.

Die einseitige Ausrichtung der argentinischen Wirtschaft um die Jahrhundertwende auf den Export von Agrarprodukten bestimmte nicht nur bleibend die Wirtschaftsstruktur und die Infrastruktur des Landes mit der Dominanz der Hauptstadtregion von Buenos Aires, sondern legte auch dauerhaft die Machtbasis der Oligarchie aus den großen estancieros und Geschäftsleuten in der Provinz und der Hafenstadt von Buenos Aires. Der Exportboom festigte die latifundio-Struktur großflächiger Produktionseinheiten und bildete die Basis einer machtvollen kleinen Elite, die den Staat zu kontrollieren wußte, die politische Macht auf nationaler Ebene zentralisierte und durch alle Wandlungen der argentinischen Gesellschaft hindurch den status quo und ihre Privilegien gegen Sozialreformen und politische Partizipationsansprüche neuer Schichten zu bewahren suchte. Der Kern dieser Schicht bestand aus 400 Familien, die in einer Reihe von Clubs und Vereinigungen eng verbunden waren. Schätzungen kommen zu dem Ergebnis, daß weniger als 2000 Personen in Argentinien soviel Land ihr Eigentum nannten, wie die gesamten Flächen von Italien, Belgien, Holland und Dänemark zusammengenommen ${ }^{11}$. Die ländliche Elite der fruchtbaren Pampas-Gebiete um Buenos Aires verschmolz mit den städtischen Geschäftsleuten und dehnte ihre Machtbasis auf die Banken und die nationale Industrie aus. Die größten Grundeigentümer kümmerten sich nicht mehr um die Verwaltung der estancias im einzelnen, sondern lebten in Buenos Aires oder oft auch in Paris als Rentiers. In Buenos Aires wachten eine Reihe von mächtigen Vereinigungen in der Zusammenarbeit mit ausländischen Kapitalgebern über ihre Interessen. Die ,Sociedad Rural Argentina“" spielt heute noch eine entscheidende Rolle im politischen Kräftespiel. Das politische System ist mit der Industrialisierung, den Militärregimen und dem Peronismus komplexer geworden und hat neue Machtfaktoren erzeugt. Obwohl der Agrarsektor nach 1945 zunehmend in seinem Anteil an dem Brottusozialprodukt abgenommen hat und Argentinien eine modernisierte, industrialisierte Gesellschaft geworden ist, liegt die entscheidende Schlüsselrolle des Agrarexports nach wie vor in der Bedeutung als wichtigste Deviseneinnahmequelle.

Weder die populistische Bewegung der ,,Unión Cívica Radical“", die der konservativen ,,liberalen" Elite in den ersten Jahrzehnten dieses Jahrhunderts Reformmaßnahmen zugunsten von Teilen der Mittelschichten abrang, noch der Peronismus der Zeit nach dem 2. Weltkrieg, noch das Militär haben je ernsthaft erwogen, eine Agrarreform durchzuführen. Die Machtbasis der Oligarchie ist niemals wirklich angetastet worden. Die Wirtschaftsstruktur

8 Vgl. Evers, a. a. O., S. 33; E. Sábato, Argentinien: Vitalität, Hoffnung und Angst, GEO-Magazin Nr. 6/1978, S. 116 ff.

9 Vgl. M. Falcoff/R. H. Dolkart, a. a. O., S. X; D. Rock, Politics in Argentina 1890-1930, Cambridge University Press 1975; M. Goldwert, Democracy, Militarism, and Nationalism in Argentina, 1930-1966, University of Texas Press 1972.

10 A. Rouquié, Pouvoir militaire et société politique en République Argentine, Fondation nationale des sciences politiques 1978: Der Autor hat seine Analyse in einem Interview in ,le monde" vom 15. 9.1978 zusammengefaßt.

11 Nachweise: D. Rock. a. a. O., S. 3. 
Argentiniens, die geringe Exportfähigkeit der nationalen Industrie und die ausländische Kontrolle der dynamischen Industriezweige geben dem Land ein geringes Maß an autonomem Steuerungsvermögen und eine erhöhte Anfälligkeit nicht nur gegenüber Schwankungen auf dem Weltmarkt, sondern insbesondere gegenüber politischen Blockierungen der Agrarproduktionen und des Agrarexportes, die Krisen in den anderen Wirtschaftszweigen und im politischen Bereich fast zwangsläufig nach sich ziehen. Das Kernproblem Argentiniens liegt sozial und politisch darin, daß eine zahlenmäßig sehr kleine Gruppe, die die wirtschaftlichen Schalthebel der Macht und den Reichtum der Nation kontrolliert, nicht bereit ist, sich zu öffnen und ihre Privilegien durch erweiterte Partizipation und wirkliche Demokratie zu gefährden ${ }^{\mathbf{2}}$.

Die argentinischen Militärregime seit 1930 traten bei aller Unterschiedlichkeit der historischen Situation und konkreten Kräftekonstellation stets als Bewahrer des status quo auf. Jedoch wäre es willkürlich, das auf konservativer Grundlage in sich heterogene Militär mit verschiedenen ideologischen und politischen Gruppierungen schlicht als Werkzeug der Oligarchie zu bezeichnen. Das Militär ist ein selbständiger Faktor mit eigenen Interessen und Vorstellungen hinsichtlich der nationalen Aufgaben. Auf den Militärputsch 1930 gegen die Gefahr der Rückkehr der Radikalen Partei an die Macht folgten fünf weitere erfolgreiche Staatsstreiche gegen Zivilregierungen der unterschiedlichsten Richtung: 1943 als Reaktion auf die alliiertenfreundliche Politik der Regierung, 1955 gegen Perón, 1962 gegen die Gefahr der peronistischen Machtübernahme, 1966 wiederum wegen eines drohenden Wahlsieges der Peronisten und schließlich 1976 gegen die ,,konstitutionelle Diktatur“ der Rechtsperonisten wegen der Unfähigkeit der korrupten Regierung mit der Wirtschaftskrise, der Guerilla, den Streiks und dem Terror fertig zu werden. Nimmt man den Zeitraum zwischen 1930 und 1973, dem Jahr der ersten Wahlen seit der ,,Argentinischen Revolution“ unter General Onganía 1966, so sind von den 16 Präsidenten 10 durch einen Militärputsch in das Amt gelangt, während 9 es auf diese Weise verloren. Das Militär regierte fast drei Viertel des genannten Zeitraums und stellte 11 Präsidenten ${ }^{13}$. Auch in den Zeiten schwacher Zivilregierungen, die ohne die Unterstützung der in die Illegalität getriebenen peronistischen Bewegung kaum wirksam regieren konnten, erwies sich das Militär als wahrer Souverän im Sinne des Begriffs bei C. Schmitt, indem es über den Ausnahmezustand entschied. Zwischen 1955 und 1966 allein provozierten die extremsten, ,,golpsita“ Teile der Streitkräfte etwa 50 politische Krisen, bis das Militär schließlich für längere Zeit die direkte Herrschaft übernahm.

Die Erhaltung des status quo ist das gemeinsame Grundziel der konservativen Streitkräfte, die die Funktion einer konservativen Massenpartei in Argentinien übernommen haben. Eine solche, die in der Lage gewesen wäre, in fairen und unverfälschten Wahlen der Radikalen Partei oder dem Peronismus wirksam entgegenzutreten, hat es in Argentinien nie gegeben. Die Meinungsverschiedenheiten hinsichtlich des Grundkonzeptes, mit dem die Krise zu lösen sei, lassen sich innerhalb des Militärs mit der Frage umschreiben: Gilt es, das ,,liberale“ Projekt des Agrarexportprogramms der Jahrhundertwende fortzusetzen und zu stabilisieren, oder soll wie 1966 in der schließlich gescheiterten „Argentinischen Revolution“ die industrielle Entwicklung auf der Grundlage einer Koalition forciert werden, die die großen multinationalen Unternehmungen, die peronistischen Gewerkschaften und die Streitkräfte einschließt ${ }^{\mathbf{1 4}}$.

\footnotetext{
12 Diese Grundthese von A. Rouquié wird auch durch die Arbeit von Evers bestätigt.

13 Evers, a. a. O., S. 39, bezieht seine Zahlen auf den Zeitraum zwischen 1930 und 1971. Die nächsten Wahlen danach fanden 1973 statt und brachten nach einer kurzen Interimspräsidentschaft Campóras Perón nach dessen Rückkehr aus dem Exil an die Macht bis zu seinem Tode 1974.

14 So A. Rouquié, le monde, a. a. O., vgl. ferner Evers, a. a. O., S. 56 ff. zu den Zielen der ,Argentinischen Revolution“ 1966.
} 
Die gegenwärtige politische Situation unter dem Militärregime ist unklar und läßt keine verbindlichen Anzeichen für eine Rückkehr zur ,,demokratischen“ Regierungsform in unmittelbarer Zukunft erkennen. Die Militärjunta, die im März 1976 die Macht in einem unblutigen Putsch übernahm, war aus den Oberkommandierenden der drei Teilstreitkräfte zusammengesetzt: General Jorge Videla, Admiral Emilio Massera und Brigadier Orlando Agosti. Die Junta erklärte in ihrem ersten Kommuniqué: ,,Angesichts des institutionellen, sozialen und administrativen Chaos, das die Republik beherrscht, hat die aus den drei Oberkommandierenden der Streitkräfte zusammengesetzte Militärjunta entschieden, die Regierung der argentinischen Nation zu übernehmen . . . Das Hauptziel ist die Wiederherstellung der unabdingbaren Werte, die für den Staat bestimmend sind . . . Die Militärjunta garantiert Leben, Besitz und Interessen der im Lande niedergelassenen Ausländer. "15

Die zunehmende innenpolitische Radikalität unter der Präsidentschaft der Nachfolgerin Peróns nach seinem Tode 1974, die sich in zahlreichen Entführungen, einigen hundert politischen Morden, Demonstrationen mit blutigem Ausgang und anderen Gewaltakten, besonders in Buenos Aires, Córdoba und in Nordwestprovinzen wie Tucumán und Catamarca äußerte, sowie die ernsten wirtschaftlichen Probleme ließen den Staatsstreich in Teilen der argentinischen Offentlichkeit wenn nicht auf vorbehaltlose Zustimmung, so doch auf Verständnis stoßen ${ }^{16}$. Bereits 1974 war der Ausnahmezustand über das ganze Land von der $\mathrm{Zi}$ vilregierung verhängt worden. Die meisten Terrorakte wurden von der rechtsextremen, offensichtlich staatliche Unterstützung genießende,,Alianza Anti-Comunista Argentina“ und linksradikalen Stadtguerillas, den hauptsächlich aus Linksperonisten bestehenden ,,Monteneros“ und der trotzkistischen „Ejército Revolucionario Popular“ verübt. Die Wirtschaftskrise erreichte 1975 bestürzende Ausmaße. Die Lebenshaltungskosten stiegen 1974 um 40,1 \% (1973 um 43,8 \%, 1972 um 64,1\%) und erhöhten sich allein von Januar bis Juli 1975 um 137,7\%. Die Auslandsverschuldung stieg auf über 8 Milliarden US-Dollar. Nachdem der ,,Sozialpakt“" zwischen Regierung, Gewerkschaften und Unternehmen zerbrochen war, erzwangen die Gewerkschaften mehrfache Regierungsumbildungen und durch einen Generalstreik im Juli 1975 eine Änderung des gegen die Inflation gerichteten Kurs der Wirtschaftspolitik. Unmittelbar vor dem Putsch standen Wirtschaft und Staatsfinanzen vor dem Zusammenbruch. Die Inflationsrate war auf $738 \%$ und die Staatsverschuldung auf 65 Milliarden DM gestiegen. Zahlungs- und Handelsbilanz waren mit jeweils 900 Millionen Dollar passiv. Für die fälligen Schuldenzahlungen in Höhe von einer Milliarde Dollar waren nur 23 Millionen an Reserven verfügbar.

Die Zahlen schwanken naturgemäß, aber Schätzungen sprechen von 1700 von Terrorgruppen Ermordeten während der 21 Monate Regierungszeit der zweiten Frau Peróns, die zum größten Teil allerdings auf das Konto der rechtsradikalen Terrorgruppen gehen ${ }^{\mathbf{1 7}}$.

Die Hauptziele der Militärjunta richteten sich gegen die Korruption und Unfähigkeit der Regierung, gegen die „,Subversion“ der linken Untergrundorganisationen und gegen die Wirtschaftskrise. Die Militärs lösten die Parlamente auf Bundes- und Provinzebene auf, setzten die Provinzgouverneure ab, verboten die Betätigung von Parteien und Gewerkschaften allgemein und bestimmte Organisationen ganz, stellten 13 Einzelgewerkschaften und den Dachverband unter Staatskontrolle und hoben das Streik- und Versammlungsrecht auf. Offentliche Bedienstete wurden der Militärgerichtsbarkeit unterstellt und Zeitungen militärisch kontrolliert. Nach Aufhebung der Hochschulautonomie wurden jede gewerkschaftli-

15 P. Fuchs, Argentinien 1976, in: S. Fronius, a. a. O., S. 132.

16 Vgl. E. Sábato, a. a. O.

17 Die Zahlen und wirtschaftlichen Daten stützen sich auf Berichte in: „,Frankfurter Allgemeine Zeitung“, „Die Zeit“, ,„le monde“, "Der Spiegel“, „,El Pais“ und „Frankfurter Rundschau“. 
che und politische Aktivität an Universitäten und Schulen unterbunden und unliebsame Intellektuelle zum Exodus gezwungen.

Die Menschenrechtsverletzungen haben unter dem Militärregime seit März 1976 unvorstellbare Ausmaße erreicht. Folter, Entführungen und Morde in Argentinien sind durch erschütternde Dokumente und Zahlenangaben durch verschiedene internationale und argentinische Organisationen und Institutionen belegt und zeugen von einer systematisch fortgeführten Verachtung elementarster Grundrechte ${ }^{\mathbf{1 8}}$.

Der von den Militärs selbst als ,,schmutzig“ bezeichnete Krieg gegen rund 20000 Guerilleros gilt offiziell als beendet. Tatsächlich scheint die Guerilla zum Erliegen gekommen sein. Jedoch hat das argentinische Militär schon oft das Ende der Guerilla verkündet, ohne daß sich dies mit den Tatsachen vereinbaren ließ. Obwohl an einer schweren Niederlage der Guerilla nicht gezweifelt werden kann, ist es ungewiß, ob die Infrastruktur der bewaffneten linken Organisationen wirklich dauerhaft zerschlagen ist. Sie befinden sich möglicherweise in einer Sammlungs- und Reorganisationsphase. Es bestehen Schwierigkeiten, die seit August 1978 stattfindenden Terrorakte und Attentate eindeutig bestimmten Gruppen zuzuschreiben, weil auch Anhaltspunkte für gewaltsame Auseinandersetzungen zwischen rivalisierenden rechtsextremen und konservativen Gruppen bestehen ${ }^{19}$.

Wenn auch der offene Terrorismus mit hemmungslosen Mitteln weitgehend ausgeschaltet wurde, so sind die Wirtschaftsprobleme kaum gelöst worden. Das Wirtschaftsprogramm des Großgrundbesitzers und Industriellen José Alfredo Martinez de Hoz ist ,liberal“ und baut auf eine strenge Blockierung des Lohn- und Gehaltsanstieges auf, die angesichts der Inflation nur unter einer Militärdiktatur durchsetzbar erscheint. Unter diesen Bedingungen konnten zwar einige Anfangserfolge in der Eindämmung der Inflation und der Verbesserung der Devisensituation erreicht werden, aber die Entwicklung läßt zunehmend an einer Erfüllung des Wirtschaftsprogramms zweifeln. Die Inflation, die 1977 zunächst auf eine Rate von $150 \%$ fiel, ist außer Kontrolle geraten und hat zu einem nie zuvor gekannten Ausmaß des Verfalls des Lebensstandards und der Kaufkraft der durchschnittlichen argentinischen Bevölkerung, vor allem der Arbeiterschaft, der Mittelschichten und der kleinen Geschäftsleute, geführt. Im Zahlungsverkehr spielen Münzen, weil wertlos, keine Rolle mehr. Im Februar 1979 wurde eine neue 50 000-Peso-Note eingeführt, um den Zahlungsverkehr zu erleichtern, für den der bis dahin höchste Wert, die 10 000-Peso-Note, nicht mehr ausreichte ${ }^{20}$.

Die Devisenrücklagen sind zwar auf mittlerweile 6 Milliarden Dollar gestiegen. Jedoch sind 125000 Beamte, Angestellte und Arbeiter aus den 700 Staatsfirmen entlassen worden, von denen viele keine Subventionen mehr erhielten oder reprivatisiert wurden. Die Industrieproduktion stagniert trotz der Verbesserung der außenwirtschaftlichen Situation, und Konkurse und Entlassungen häufen sich, während ausländische Investitionen sehr zurückhaltend sind und wichtige Konzerne wie General Motors und Fiat ihre Produktion in andere Länder verlagern. In dieser Situation spitzt sich der Konflikt mit den Gewerkschaften immer mehr zu. Ein Höhepunkt war der illegale Lohnstreik der Eisenbahner in Buenos Aires im November 1978. Obwohl das Militär mit dem Einsatz von Soldaten drohte und 50 Gewerkschaftsführer verhaftete, mußte es wegen der Gefahr eines nationalen Aufruhrs nachgeben, der bei den zu erwartenden Todesfällen bei einem harten Durchgreifen provoziert worden wäre.

$18 \mathrm{Vgl}$. Amnesty International, Bericht über eine Mission nach Argentinien 6.-15. Nov. 1976, Wien 1977. Die OAS-Menschenrechtskommission wird ihren Bericht im Frühjahr 1980 vorlegen, vgl. FAZ 24. 9. 1979.

19 le monde vom 29. 12. 1978.

20 Die Zeit Nr. 20 vom 11. 5. 1979 mit einem Situationsbericht von W. A. Wiegand. 
Uber die politische Zukunft ist das Militär zerstritten. Uneinigkeit besteht vor allem in der Frage, wie der Widerstand der Arbeitnehmer gegen die Wirtschaftspolitik zu behandeln ist. Tiefe Meinungsverschiedenheiten offenbaren sich auch in der Frage, wann und unter welchen Bedingungen Zivilisten an die Ausübung der Regierungsfunktionen zu beteiligen sind. Videla ist 1978 aus der Junta ausgeschieden und übt das Amt des Staatspräsidenten aus, um die Rolle einer übergeordneten Instanz zu den Befehlshabern der drei Teilstreitkräfte einzunehmen. Jedoch ist es unklar, wer den politischen Kurs bestimmt, da die Kompetenzen zwischen dem Präsidenten und der Junta, in die der Vertraute Videlas, General Robert Viola, für das Heer eingerückt ist, nicht abgegrenzt sind. Vorläufig scheint Videla bis 1984 im Amt bleiben zu wollen und einen nicht näher erläuterten Weg der ,,renovación“, der Erneuerung der politischen Parteien einzuschlagen. Auch die Pläne des Heeres lassen keinen Zweifel daran, daß dieser vage Prozeß auf längere Zeit die Vorherrschaft des Militärs einschließt. In der Luftwaffe und in der Marine treffen die Vorschläge Videlas und des Heeres auf harte Opposition. Die Luftwaffe strebt ein möglichst langes Verbleiben der Militärs in der Regierung an und hat 1978 einen Plan vorgelegt, der für weitere 10 Jahre parteipolitische Aktivitäten,,einfrieren“" will und dann die Gründung einer von den Streitkräften dominierten ,,offizialistischen“ Partei vorsieht. Das Heer dagegen spricht von der baldigen Gründung einer ,,Movimiento de Opinión Nacional", die nicht den Charakter einer von dem Militär beschirmten Partei haben soll. Die Marine will den „,Prozeß der nationalen Reorganisation“ mit der Gründung einer politischen Partei unter direkter militärischer Vormundschaft durchführen $^{21}$.

In der Uneinigkeit der Streitkräfte über eine für die unmittelbare Zukunft kaum zu erwartende ,Rückkehr zur Demokratie“ ist das Wiederauftauchen der politischen Parteien mit bestimmten Forderungen kaum ein relevanter Faktor. Als meist korrupte, personalisierende und politisch unfähige Cliquen ohne ernsthafte Verankerung in der Bevölkerung sind diese kaum eine überzeugende Alternative. Als stärkste politische Kraft sind trotz der Zersplitterung wohl immer noch die Uberreste der peronistischen ,Frente Justicialista de Liberacion“ zu bezeichnen, weil sie in den Gewerkschaften eine Stütze finden. Im Bündnis mit dieser Gruppe scheint sich inzwischen der ehemalige Marinechef Massera, der im September 1978 aus der Junta ausschied und im Dezember ein Attentat überlebte, in der argentinischen Offentlichkeit als Gegenspieler Videlas mit einer scharfen Kritik an den sozialen Folgen der Wirtschaftspolitik zu entwickeln.

Prognosen über die zukünftige politische Entwicklung Argentiniens sind nicht möglich. Sie hängt jedoch entscheidend von dem Konflikt zwischen den Gewerkschaften und der Militärregierung einerseits und den Konflikten zwischen den rivalisierenden Fraktionen im Militär selbst andererseits ab. Der alte peronistische Gewerkschaftsbund ,CGT"steht nach wie vor unter militärischer kommissarischer Verwaltung. Aber es sind Bestrebungen vorhanden, Gewerkschaftsgruppen zu einem neuen Dachverband zusammenzuschließen, denen der argentinische Innenmister General Harguindeguy mit Berufung auf die „Prinzipien der Militärjunta" entgegenzutreten versucht hat ${ }^{22}$. Auf der anderen Seite ist im September 1979 ein Machtkampf im Heer ausgebrochen, als Viola den weiter rechts stehenden Kommandeur des dritten Armeekorps in Córdoba, Menendez, absetzte. Dieser verschanzte sich in einer Militärschule gegen eingesetzte Luftlandetruppen und war auch nach Zusicherung von freiem Geleit nicht bereit, zu einer Besprechung mit der Junta nach Buenos Aires zu reisen. Menendez warf Viola vor, Personenkult zu betreiben, den Zusammenhalt des Heeres zu untergra-

21 FAZ vom 31. 10. 1978.

22 FAZ vom 21. 4. 1979. 
ben und ,,durch Nachlässigkeit der marxistischen Subversion aufs neue Chancen geboten“" zu haben ${ }^{23}$.

Daß unter diesen Bedingungen Probleme des Föderalismus in Argentinien nicht im Mittelpunkt des Interesses stehen, ist offensichtlich. Jedoch ist historisch das „Provinzproblem“ in Argentinien exemplarisch geeignet, die allgemeinen Schwierigkeiten des Verfassungsrechtes und der politischen Institutionen in diesem bis 1930 oft mit Australien, Kanada und mit der zugehörigen Úbertreibung selbst mit den USA verglichenen Landes zu beleuchten.

\section{Der historische Hintergrund des argentinischen Föderalismus und das „,Provinzproblem“}

Die politische Einteilung Argentiniens in Provinzen und Territorien (,,gobernaciones") hat sich im Laufe der Geschichte immer wieder verändert. Zur Zeit der Annahme der Verfassung von 1853 gab es 14 Provinzen. Besonders in den fünfziger Jahren dieses Jahrhunderts erhielten eine Reihe von Territorien mit einigen Grenzverschiebungen den Status von Provinzen. Heute ist Argentinien in 22 Provinzen zuzüglich der Territorien Feuerland, Antarktis und Südatlantische Inseln und dem Bundesdistrikt, der Hauptstadt Buenos Aires, aufgeteilt. Der historische Keim für die Jahrzehnte des Bürgerkrieges und der Anarchie, die der Unabhängigkeitserklärung 1816 in der Auseinandersetzung zwischen ,,federales“ und ,,unitarios “, Anhängern des Bundesstaates und denen des Einheitsstaates, folgten und erst 1880 mit der Erklärung der Hafenstadt Buenos Aires zur Bundeshauptstadt wirklich endeten, wurde mit der Neuorganisation des spanischen Vizekönigtums im 18. Jahrhundert gelegt. Bis ins 18. Jahrhundert hinein waren die spanischen Kolonien von Río de la Plata von dem Vizekönigtum in Perú abhängig" ${ }^{24}$ Die Gouverneure der ,,corregimientos“ wurden von der Krone ernannt, während die städtische Verwaltung in den Händen der ,,calbildos“, der Stadträte, lag. Schon die frühe Besiedlungsgeschichte läßt eine $\mathrm{Zweiteilung} \mathrm{des} \mathrm{Landes} \mathrm{erkennen.} \mathrm{Ei-}$ nerseits konzentrierte sich die Kolonisierung auf das ailantische Küstengebiet mit der Gründung von Buenos Aires im Jahre 1530. Auf der anderen Seite wurden als eine Folge der Ausdehnung der Eroberungen von Perú und Chile die landesinneren Regionen mit der sporadischen Gründung einer Kette von Städten besiedelt. Das Vizekönigtum von Río de la Plata wurde mit Sitz in Buenos Aires 1776 errichtet. Der Grundstein für den späteren Konflikt zwischen Buenos Aires und den Provinzen im Hinblick auf den Grad der jeweiligen Autonomie wurde 1782 gelegt. Das Vizekönigtum wurde in sieben Provinzen (,,intendencias“), zwei militärische Regierungsbezirke und die beaufsichtigende Regierung von Buenos Aires gegliedert.

Das typische spanische Muster der ,,municipios“, Städte, die die gesamte ländliche Umgebung kontrollierten, erzeugte regionale politische Systeme, die lokale Gouverneure ein erhebliches Maß an Autonomie verschaffte ${ }^{25}$. Die Unabhängigkeitsbewegung zu Anfang des 19. Jahrhunderts und die Entstehung des argentinischen Nationalbewußtseins verdeckten in der Auseinandersetzung mit äußeren Feinden zunächst die inneren Spannungen. Die Unabhängigkeit zerstörte den spanischen Versuch, den südlichen Teil Südamerikas über zentralistische Regierungsformen unter direkte Kontrolle zu bringen, und regionale ,,caudillos“ konnten ihre Machtbasis in den wichtigsten Städten und den alten Domänen sicherstellen.

\footnotetext{
23 FAZ vom 1. 10. 1979.

24 Vgl. Constitutional Chronology, Argentina, in: A. P. Blaustein/G. H. Flanz, Constitutions of the Countries of the World, New York 1974, S. $1 \mathrm{ff}$.

25 R. H. Dolkart, a. a. O., S. 166.
} 
Bis zum Ende des 19. Jahrhunderts war Argentinien keine einheitliche Nation, sondern eher eine lockere Konföderation, in der jede Provinz unter den regionalen caudillos ein großes $\mathrm{Maß}$ an Unabhängigkeit hatte ${ }^{26}$. Wahre Glücksritter und Bandenchefs stützten sich auf regionale Milizen und Gauchoheere, um die sich immer deutlicher abzeichnende Tendenz der Hegemonie von Buenos Aires, der fruchtbaren Küstenregion mit der gleichnamigen Hafenstadt, abzuwehren. Der steigende Bedarf auf dem Weltmarkt nach Agrarprodukten im 19. Jahrhundert brachte Argentinien eine günstige Lage, von der besonders die Provinz und die Stadt Buenos Aires beim Exporthandel und der Verbindung mit Europa profitierte. Ein unumkehrbarer Prozeß der zunehmenden Konzentration der wirtschaftlichen Entwicklung und des Reichtums in der Region von Buenos Aires und der wirtschaftlichen und politischen Unterordnung der anderen, in ihrer Bedeutung absteigenden Provinzen wurde eingeleitet. Buenos Aires hatte den einzigen Hafen Argentiniens, den alle Waren, die ins Land kamen oder es verließen, passieren mußten. Durch das Monopol der Zollerhebung, des Bankwesens und der Geldemission erfuhr die Stadt einen schwindelerregenden Aufstieg auf Kosten der peripheren Provinzen. Der lateinamerikanische Historiker Eduardo Galeano macht darauf aufmerksam, daß fast alle Einkünfte von Buenos AiresihrenUrsprung in den Zollgebühren hatten, die der Hafen unrechtmäßig zu seinem eigenen Nutzen verwendete, und von denen mehr als die Hälfte zur Kriegsführung gegen die Provinzen aufgewendet wurde ${ }^{27}$.

Die berüchtigte Diktatur Juan Manuel de Rosas (1829-1832; 1835-1852), dessen militärische Auseinandersetzungen mit den Provinz-caudillos der spätere argentinische Präsident Sarmiento (1868-1874) in seinem Werk ,Facundo“ beschrieben hat, ordnete die Provinzen der Hafenstadt auch politisch unter. Im Lichte der geographischen, wirtschaftlichen, sozialen und politischen Realitäten erwiesen sich die Durchsetzung föderaler Prinzipien in der Verfassung von 1853 und die Abtrennung von Buenos Aires als Bundeshauptstadt von der gleichnamigen Provinz, die nun von La Plata aus regiert wurde, im Jahre 1880 nach einer erneuten militärischen Auseinandersetzung in Wahrheit als ein Pyrrhussieg ${ }^{28}$.

Es wäre jedoch verkürzt, wenn man die soziale Dimension der Auseinandersetzungen in der Epoche der argentinischen Anarchie übersehen würde und die Kämpfe zwischen Linientruppen aus Buenos Aires und Lanzenreitergruppen aufbegehrender Gauchos, von denen es hieß, sie seien ,,zweifüßige Tiere“ mit ,,perversem Charakter“, deren Blut als „,Dünger“ zum ,Nutzen des Landes verwendet werden muß“29, lediglich als Interessengegensätze zwischen der Oligarchie in Buenos Aires und den regionalen caudillos interpretieren würde. Die ,Modernisierung“ Argentiniens war auch mit der Entrechtung und sozialen Verelendung breiter Teile der Provinzeinwohnerschaft verbunden, deren chronische Armut Rebellion gegen die reichen Großgrundbesitzer und Kaufleute hervorrief.

Die Verfassungsgeschichte Argentiniens im engeren Sinne hat nach den Kapitularakten von 1810 eine Reihe von wichtigen Gesetzen und Verfassungsprojekten hervorgebracht, die zwar nicht gänzlich ohne Einfluß auf die spätere Gestalt des Verfassungsdokumentes geblieben sind, meist aber Entwurf oder Papier mangels Konsens zwischen den Provinzen blieben und deshalb hier vernachlässigt werden können ${ }^{30}$. Bis 1852 gab es keine ernsthaften Versuche, Argentinien mit verfassungsrechtlichen Mitteln zu einen. Nachdem Rosas von General Urquiza geschlagen wurde, legte das Abkommen von San Nicolás in diesem Jahr das föderalistische Regierungssystem fest. Die Absprache bot das Fundament für die Verfassung von

26 Vgl. das Schrifttum in Anm. 6, 9 und 10.

27 E. Galeano, Die offenen Adern Lateinamerikas, 2. Aufl., Wuppertal 1976, S. 209, $214 \mathrm{ff}$.

28 Dolkart, a. a. O., S. 165

29 Galeano, a. a. O., mit Zitaten von führenden argentinischen Staatsmännern der Zeit.

30 Vgl. P. Ramella, Die Entwicklung des Verfassungsrechts in Argentinien, 18 JOR NF (1969), S. 649 ff., übersetzt aus dem Span. von W. R. Koelling. 
1853, fand die Zustimmung der Provinzen, nicht aber von Buenos Aires. Buenos Aires lehnte auch die Ratifikation dieser nach nordamerikanischem und chilenischem Vorbild geschaffenen und von Alberdi den argentinischen Verhältnissen angepaßten Verfassung ab. Es erklärte sich zum unabhängigen Staat außerhalb der Argentinischen Konfederation und wehrte ein Gesetz ab, das das Stadtgebiet von Buenos Aires zur Hauptstadt erklären und als Bundesgebiet der Aufsicht der Gesetzgebung und des Präsidenten der Konfederation unterstellen wollte.

Militärische Auseinandersetzungen zwischen der Konfederation und Buenos Aires, teilweise wegen Zollstreitigkeiten, führten zur Revision der Verfassung im Jahre 1860. Die Änderung stärkte die Rechte der Provinzen, versäumte es aber, die Fragen der Hauptstadt und der Finanzquellen der Nation zu regeln. Man behalf sich damit, Buenos Aires zur vorläufigen Hauptstadt zu erklären und der Bundesregierung bis 1866 Vollmachten zur Finanzdekkung einzuräumen.

Konsolidiert wurde die politische Einheit erst wirklich in dem Zeitraum zwischen 1868 bis 1880. Kaum mit Buchstaben und Geist der Verfassung zu vereinbaren, wurde de facto der Händel zwischen Buenos Aires und den Provinzen mit Hilfe des ,,unicato“, des Einpersonenregimes, beigelegt ${ }^{31}$. Die Wahlen wurden von der ,Partido Autonomista Nacional“"manipuliert. Die Präsidenten kontrollierten Politik und Verwaltung fast vollständig. Abhängige Provinzchefs stellten die Integration lokaler und regionaler Interessen in die Politik der Zentralregierung sicher. Wirkten das System der Korruption und persönlicher Gunstbeweise nicht, so ließ sich provinzieller Widerstand leicht mit dem Mittel der Intervention durch die Zentralgewalt beseitigen. Der Präsident hatte keine Schwierigkeiten, mißliebige regionale Behörden abzusetzen, neue Personen einzusetzen und auf Bundesebene die Senatsmehrheit zu verändern. 1880 transformierte ein Gesetz Argentiniens aufblühende $\mathrm{Ha}$ fenstadt zur dauerhaften Hauptstadt und zum Bundesgebiet.

Das ,Provinzproblem“ und die immer offensichtlicher werdende Dominanz der Hauptstadtregion wurden in den folgenden Jahrzehnten des in Lateinamerika beispiellosen Aufschwungs Argentiniens von 1880 bis zur Weltwirtschaftskrise verdrängt. Die argentinische Wirtschaft wurde einseitig auf den Export von Agrarprodukten vor allem für den britischen Markt ausgerichtet und erhielt Industrieerzeugnisse und Kapital im Austausch. Die Provinzen erhielten ihren Anteil an dem Reichtum, jedoch waren es die Provinz und die Stadt Buenos Aires, die am meisten von der wirtschaftlichen Entwicklung profitierten. Auch die Einwanderung von etwa 3,5 Millionen Menschen, hauptsächlich aus Italien und Spanien, was einer Erneuerung der demographischen Struktur gleichkam, kam in erster Linie der Hauptstadtregion zugute. Später sollte sich auch die Industrialisierung auf diesen Raum konzentrieren. Heute läßt sich das Entwicklungsgefälle zwischen der Metropole und dem Hinterland durch kreisförmig um Buenos Aires herumlaufende Ringe veranschaulichen. Die Bevölkerungsbewegung verläuft einseitig von den rückständigen ländlichen Zonen des Westens und Nordens in das östliche wirtschaftliche Kerngebiet, wo Hunderttausende ihre Heimat und Hoffnung in kaum beschreiblichen Slums gefunden haben. In dem Großraum von Buenos Aires leben 33,7 \% der Bevölkerung, das sind mehr als 8 Millionen. Dagegen sind große Provinzen wie Chubut und Santa Cruz fast menschenleer. Die Hauptstadtregion beherbergt $40 \%$ aller Industriebetriebe, $53 \%$ aller Beschäftigten und erwirtschaftet $56 \%$ der Gesamtproduktion. Die Zahl der Ärzte ist relativ wesentlich höher als im übrigen Land und ebenso trifft dies für die Bankeinlagen zu. Die Kindersterblichkeit liegt erheblich unter dem nationalen Durchschnitt. Auch andere Daten zeugen von der Dominanz der Haupt-

31 Vgl. Dolkart, a. a. O., S. 172 ff.; D. Rock, a. a. O. 
stadtregion, die faktisch so überwältigend geworden ist, daß eine der Kernfragen jeder föderalen rechtlichen Regelung unausweichlich das Problem des Ausgleichs der ungleichen Finanz- und Ressourcenverteilung mit eigener Entscheidungsbefugnis der Provinzen ist ${ }^{\mathbf{3 2}}$. Das heutige Verhältnis von Hauptstadt und dem ,,anderen Argentinien“ beschreibt Sigrid Fronius in anschaulicher Weise: „Die Hauptstadt Buenos Aires heißt in der Provinz ,el Pulpo', die Krake. Mit ihren Fangarmen hält sie die Provinzen Argentiniens umklammert und saugt aus ihnen allen Reichtum, der dort produziert wird. Eisenbahn und wichtige Verkehrsstraßen führen direkt nach Buenos Aires, wo die Rohprodukte entweder im Hafen nach dem Ausland verschifft oder in den Fabriken des Industriegürtels verarbeitet werden. Zu höheren Preisen als in der Hauptstadt tauchen dann die verschiedenen Produkte in den Krämerläden des Dorfes oder in der Provinzhauptstadt wieder auf . . . Vereinzelt liegen in diesen über tausend Kilometer von der Hauptstadt entfernten Provinzen Bergwerke, stehen Bohrtürme und Elektrizitätswerke, deren Leitungen direkt zur Hauptstadt führen und das Land, das sie durchqueren, ohne Kanalisation und Elektrizität lassen. All diese Provinzen weisen Monokultur auf. Die Besitzer der Ländereien sitzen in Buenos Aires. Solange das Geschäft gutgeht, pflanzen sie Tee, Baumwolle, Tabak oder Zucker in großen Mengen. Doch die Gewinne fließen nicht in die Provinz zurück, sie finanzieren vielmehr die neuen Fabriken, die um Buenos Aires wie Pilze aus dem Boden schießen. Der Provinzpolitiker ist eine Marionette der Zentralregierung, der Provinzbourgeois ein Bittsteller bei den Kreditinstituten und Banken der City von Buenos Aires, und auch der Gewerkschaftssekretär kann nur versuchen, sich so gut es geht gegen die reichen Bürokraten aus der Großstadt zur Wehr zu setzen. Auch er muß ständig nach Buenos Aires reisen. “'33

Nach der ,Argentinischen Revolution“ 1966 unter General Onganía stritten sich argentinische Verfassungsjuristen, ob Argentinien noch als föderaler oder materiell schon als unitarischer Staat zu bezeichnen sei ${ }^{34}$. Wenn dieser Kontroverse mit Rücksicht auf die realen Verhältnisse überhaupt eine Bedeutung zukam, so war sie jedenfalls längst verspätet. Formal mag die Verfassung von 1853 mit ihren späteren Änderungen Argentinien eine bundesstaatliche Gestalt gegeben haben. Im politischen Prozeß und in der Verfassungswirklichkeit haben die Provinzen im Verhältnis zu Buenos Aires nach 1880 als autonome Entscheidungseinheiten bald an Bedeutung verloren ${ }^{35}$. Die regierende Partei in Buenos Aires hat stets mit verfassungsrechtlichen und besonders mit außerverfassungsrechtlichen Mitteln ihre Herrschaft auch in den Provinzen sichergestellt.

Die Zentralisierung der politischen Macht ergab sich fast zwangsläufig daraus, daß die Provinzen in ein nationales Markt- und Produktionssystem integriert wurden, welches das historische Muster der ständigen Revolten gegen die Hegemonie von Buenos Aires in profitable Komplizenschaft und Zusammenarbeit der provinziellen Oligarchie mit der Zentralregierung verwandelte, um den Status quo zu erhalten. Wahlen in den Provinzen wurden systematisch verfälscht und mit Gewalt manipuliert. Auf der anderen Seite übten auch einflußreiche provincianos, Mitglieder der Provinzoligarchie, Regierungsfunktionen in Buenos Aires aus, etwa im Senat oder dadurch, daß sie Positionen auf Kabinettsebene oder darunter erhielten.

Das ,Provinzproblem“ und der Abstieg der Autonomie der Provinzen erregte das öffentliche Interesse wieder in den Jahren zwischen 1930 und 1943. Faktisch bestand kein Zweifel daran, daß Argentinien ein unitarischer Staat geworden war. Allerdings zeigte sich bald, daß

32 Vgl. Waldmann, a. a. O., S. 16; Dolkart, a. a. O., S. 181 ff. mit statistischen Daten.

33 Fronius, a. a. O., S. 44 f.

34 Nachweise bei Evers, a. a. O., S. 90.

35 Vgl. Dolkart, a. a. O.; besonders erhellend im Hinblick auf die realen Strukturen ist die Arbeit von Rock, a. a. O. 
die Substanz des „Provinzproblems“ sich verändert hatte und nicht mehr wie im 19. Jahrhundert mit den Schlagworten Föderalismus versus Zentralismus zu erfassen war. Einerseits schließt das Föderalismusproblem in Argentinien nicht mehr den Gegensatz Zentralstaat oder Bundesstaat versus Gliedstaaten oder den Antagonismus zwischen Zentralgewalt und Provinzgewalt ein, sondern setzt die Zentralgewalt synonom mit der Bundeshauptstadt gegen die Gewalt und Rechte der Provinzengesamtheit ${ }^{36}$. Andererseits hat die Entwicklung zunehmend die soziale Dimension des „Provinzproblems“ in den Vordergrund treten lassen. Der Verfall der Provinzen und der Reichtum der Hauptstad that mit zunehmender Krise den Gegensatz zwischen arm und reich, zwischen den ,,descamisados“ und ,,campesinos“, dem ,Volk“, und der städtischen und landbesitzenden Oligarchie bestimmend werden lassen.

Der Aufstieg des Peronismus hat letztlich hierin seine sozialen Wurzeln. Nach der Revolution 1943 wurden die Provinzen bis zur Wahl 1946 in direkter Abhängigkeit von den durch das Militär kontrollierten Bundesbehörden gehalten. Die verfassungsmäßige Wiederherstellung der Provinzautonomie nach der Wahl Peróns 1946 war bloßes Papierwerk, denn die Peronisten unterdrückten jede Opposition und stellten ihre Herrschaft auch in den Provinzen sicher, die mit peronistischer Wahlpropaganda nicht zu gewinnen waren. Bis 1949, als die peronistische Verfassung verabschiedet wurde, zeichneten sich die Provinzverfassungen durch eine gewisse Vielfalt aus, soweit dies eben nach den Prinzipien der Bundesverfassung erlaubt war. Die Verfassung von 1949 unterschied sich hinsichtlich der bundesstaatlichen Ausgestaltung kaum von dem Text der alten Verfassung. Aber die Provinzen wurden gezwungen, ihre Verfassungstexte neu zu schreiben, um sie in Ubereinstimmung mit der neuen Verfassung zu bringen. Peróns ,Neuer Föderalismus“ bedeutete totale Kontrolle über die Provinzen mit dem Zweck, statt den Schein regionaler Autonomie aufrechtzuerhalten, die Lebensbedingungen im ganzen Land zu verbessern ${ }^{37}$.

Tatsächlich zeigten sich Änderungen in der Stagnation der Provinzentwicklung, die mit einer Umverteilungspolitik erreicht wurde, die die Dominanz von Buenos Aires nicht mehr vorbehaltlos anerkannte. Die Reformen verlangsamten sich aber zu Beginn der fünfziger Jahre mit den zunehmenden wirtschaftlichen und innenpolitischen Schwierigkeiten, die 1955 zum Sturz Peróns führten. Der Aufstand gegen Perón begann nicht zufällig in Córdoba, in der Provinz.

Wenn auch die Zeit nach 1955 wesentlich durch den Gegensatz zwischen dem Militär und den Peronisten ${ }^{\mathbf{3 8}}$, vor allem in den mächtigen, von Perón politisch institutionalisierten peronistischen Gewerkschaften, die nicht mit Vorstellungen über europäische Arbeitnehmerorganisationen begriffen werden können, geprägt ist, so ist auch das Landesinnere zu einer eigenen politischen Größe mit gesamtstaatlicher Bedeutung geworden ${ }^{39}$. Das Gefühl der $\mathrm{Zu}$ rücksetzung in der Provinz hat sich nach der Machtergreifung durch das Militär 1966 in Aufständen geäußert, zuerst im „Córdobazo“, in Córdoba im Mai 1969, dann in späteren Revolten in Provinzstädten wie Mendoza oder Tucuman. Die Aufstände wurden militärisch niedergeschlagen. Aber sie signalisierten auch das Scheitern des ehrgeizigen Projektes der „Argentinischen Revolution“40. General Onganía mußte zurücktreten und wurde durch einen anderen Militär ersetzt. Doch auch General Levingston mußte im März 1970 nach einem Aufstand in der Provinz, der ,Viborazo“, abtreten.

36 V. H. Martinez, Federalismo y política centralista, in: J. C. Agulla (Hrg.), Federalismo y centralismo, Buenos Aires 1967, S. 75. 37 Dolkart, a. a. O., S. $194 \mathrm{f}$.

38 Besonders instruktiv der knappe Aufsatz von J. C. M. Ogelsby, Argentinien zwischen Militärherrschaft und Peronismus, EuropaArchiv 1973, S. $525 \mathrm{ff}$.

39 Vgl. Fronius, a. a. O., S. $50 \mathrm{ff}$.

40 Die Analyse der "Argentinischen Revolution“ ist Gegenstand der Arbeit von Evers. 
Die Rückkehr zur Zivilregierung 1973 mit dem Wahlsieg der Rechtsperonisten bedeutete keine Rückkehr, oder besser: keinen Beginn der Respektierung der Provinzautonomie. Das Mittel der Intervention war ausreichend, um einen der Zentralregierung unliebsamen linksperonistischen Provinzgouverneur loszuwerden. Seit 1974 wurde unter Ausnahmezustand regiert, den die Militärs nach ihrem Putsch 1976 zur Zerschlagung der Guerilla und der Terrorgruppen beibehielten.

Von diesem hier nur angedeuteten Hintergrund des „Provinzproblems“ in Argentinien in seinem Wandel von der historischen Ausgangssituation im 19. Jahrhundert bis zur heutigen tiefgreifenden Krise der argentinischen Gesellschaft ist die verfassungsrechtliche Ausgestaltung des Bundesstaatsprinzips in der Verfassung von 1853 mit den späteren Änderungen zu betrachten. Zu dieser Verfassung, die angeblich auch heute noch formell in Kraft sein soll, kehrte eine verfassungsgebende Versammlung 1957 im wesentlichen zurück, nachdem die peronistische Verfassung abgeschafft worden war.

\section{Die Regelung des Föderalismus in der formellen Verfassung}

Die „,Constitución De La Nación Argentina“, ,,sancionada par el Congreso General Constituyente el $1^{\circ}$ de mayo de 1853 , reformada y concordada par la Convención Nacional ,Ad hoc' el 25 de septiembre de 1860 y con las reformas de las convenciones de 1866, 1898 y 1957“41, greift für lateinamerikanische Verhältnisse auf eine vergleichsweise lange Verfassungstradition zurück. Die formelle Verfassung hat jedoch kaum je den realen politischen Prozeß zu regeln vermocht. Auch läßt sich die Funktionsweise der Institutionen, die die Urkunde vorsieht, auch nicht annähernd aus dem Verfassungstext ablesen ${ }^{42}$. Wenn man heuristisch zwischen formeller, materieller Verfassung und politischer Realverfassung unterscheidet ${ }^{43}$, so sind in Argentiniens Verfassungsgeschichte stets die Strukturen und Regeln der letzteren in großer Diskrepanz zur ersten bestimmend. Im Schrifttum ist meist davon die Rede, daß die Periode zwischen 1880 und 1928, im Gegensatz zu der andauernden Krise danach, Jahre der politisch-institutionellen Stabilität gewesen seien ${ }^{\mathbf{4}}$. Auch nach der Einführung des Wahlrechts für Männer argentinischer Staatsbürgerschaft 1912 bedeutete diese Stabilität keine demokratischen Verhältnisse. Die Herrschaft der Oligarchie stützte sich trotz der liberalen Verfassungsurkunde auf ein System der Korruption, persönlichen Gunstbeweise, Wahlfälschung und Manipulation, nicht zuletzt, um mit Hilfe des Anscheins demokratischer Zustände ausländische Investitionen nicht durch eine Wiederholung der Schreck-

41 Der spanische Text ist mit einerfranzösischen Übersetzung veröffentlicht in: Corpus Constitutionnel, I, Leiden 1972, S. 365 ff.; englische Ubersetzungen finden sich in Blaustein/Flanz, a. a. O., und ferner in: A. J. Peaslee, Constitutions of Nations, Vol. IV, Netherland 1970.

42 Die beste juristische Einführung in das argentinische Verfassungsrecht in deutscher Sprache ist die Ubersetzung der Arbeit von Ramella, a. a. O., des Dekans der Juristischen Fakultät der Katholischen Universität von Cuyo(San Juan), Argentinien. Eine kritische Analyse des Verfassungsrechtes leistet insbesondere J. R. Vanossi, République Argentine, L'évolution institutionnelle depuis 1970, in: Corpus Constitutionnel, a. a. O. Zur Verfassungskontrolle vgl. H. Fix-Zamudio, Verfassungskontrolle in Lateinamerika, 25 JOR (1976), S. 649 ff.; ferner P. G. Snow, Judges and Generals: The Role of the Argentine Supreme Court during Periods of Military Government, 24 JOR (1975), S. 609 ff.

43 Diese Verwendung des Verfassungsbegriffs im Sinne der "Staatsverfassung“ ist im ,ostrechtlichen“ Schrifttum vor allem von B. Meissner in Anlehnung an H. Heller entwickelt worden, vgl. B. Meissner, Die Rechtsstellung der Kommunistischen Partei der Sowjetunion, Jahrbuch für Ostrecht 1961, Bd. II/2, S. 7 ff. (10). Diese Unterscheidung ist in einem heuristischen Sinne hilfreich, wenn die Diskrepanz zwischen dem formellen Verfassungsrecht und der Wirklichkeit besonders groß ist und die formelle Verfassung ein trügerisches Bild von den wirklich geltenden Regeln vermittelt. Eine Diskussion der Begriffe „,Staatsform“, „,Regierungsform“, ,,Regierungssystem“ findet sich neuerdings bei G. Brunner, Entwurf einer Verfassungstypologie sozialistischer Staaten, ROW 1978, S. 49 ff. Grundsätzliche Einwände gegen die juristischen staatstheoretischen Zentralbegriffe erhebt N. Luhmann, Politische Verfassungen im Kontext des Gesellschaftssystems, Der Staat 1973, S. 1 ff. und 165 ff. Zur Verfassungsvergleichung: H. Krüger, Stand und Selbstverständnis der Verfassungsvergleichung heute, VRU 1972, S. 5 ff.

44 Etwa Waldmann, a. a. O., S. 35. 
nisse während der Tyrannei de Rosas zu entmutigen. Analysen der politischen Institutionen und der konkreten Machtverteilung in der Regierungszeit der Radikalen nach 1916 bis zum Sturz Yrigoyens durch das Militär im Jahre 1930 zeigen, daß die Hegemonie der Oligarchie trotz der Veränderungen Argentiniens in keiner substantiellen Weise geschwächt wurde ${ }^{45}$. Nach 1930 ist das Verfassungsrecht über die Zwischenphase des Peronismus (1946-1955) hinaus durch den Aufstieg des Militärs gegenüber der politischen Realverfassung bedeutungslos.

Die Militärregime haben juristisch stets Hochverrat nach Art. 22 der Verfassung begangen, und die Befugnisse, die sich die Generäle angemaßt haben, gehen weit darüber hinaus, was Art. 23 dem Staatspräsidenten im Notfall einräumt. Die Militärs sind jedoch stets auch als Verteidiger der demokratischen Verfassung aufgetreten, haben ihre Herrschaft als Ubergangsphase zur Demokratie deklariert und versucht, die Verfassungsformen formal soweit aufrechtzuerhalten, wie sich dies mit ihren Zielen und ihrer Politik vereinbaren ließ. De jure ist die verfassungsrechtliche Lage durch die Herrschaft der ,,De-facto-Regierungen“, durch die Uberlagerung des Verfassungsrechtes durch Revolutionsstatute und Militärdekrete und durch die die Faktizität der Staatsstreiche teilweise legitimierende Rechtsprechung des Obersten Gerichtshofes so unübersichtlich geworden, daß argentinische Juristen sich eine Lösung nur noch durch eine neue verfassungsgebende Versammlung versprachen ${ }^{46}$. Eine solche Versammlung, für die es zur Zeit keine Aussichten gibt, könnte vielleicht eine bereinigte neue formelle Verfassung verabschieden, die Grundproblematik des argentinischen Verfassungsrechtes liegt jedoch nicht in der juristischen Konstruktion, sondern in der politischen Realverfassung, in der politischen Kultur und der sozioökonomischen Krise Argentiniens.

Dies gilt es im Gedächtnis zu behalten, wenn die Grundmerkmale der formellen Verfassung mit den Begriffen liberal, repräsentativ und föderal, mit einer starken Betonung der Präsidialdemokratie gekennzeichnet werden. Viele Artikel sind wörtlich der USA-Verfassung entnommen.

Die argentinische Verfassung enthält in ihrem ersten Teil eine Reihe von liberalen und sozialen Grundrechtserklärungen. Sie garantiert das Recht auf Arbeit, des Handels und der Petition, gewährleistet die Freizügigkeit, die freie Ein- und Ausreise, die Freiheit der Presse, des Eigentums, der Vereinigung, der Religion und Erziehung. Aus der peronistischen Zeit sind die Schutzbestimmungen zugunsten der Arbeiter und der Gewerkschaften sowie die Wohlfahrtsregelungen übernommen worden (Art. 14). Die Sklaverei ist verboten, was auf eine frühe politische Entscheidung in der Verfassungsgeschichte zurückgreift, die Gleichheit vor dem Gesetz ist verkündet. Die individuellen Sicherungen des Art. 18 sind unmittelbar von der USA-Verfassung und dem englischen Habeas-Corpus-Prinzip beeinflußt. Das spezifische Sicherungsmittel ist hier das argentinische, von den Anwaltskonferenzen, der Rechtsprechung und der Literatur entwickelte Amparo-Verfahren ${ }^{47}$.

Art. 36 legt ein Zweikammer-System fest. Als gesetzgebende Gewalt besteht der Kongreß aus der Abgeordnetenkammer und dem Senat. Auf Bundesebene werden die Abgeordneten alle vier Jahre direkt gewählt. Der Senat setzt sich aus je drei Mitgliedern sämtlicher Provinzen zusammen. Zwei dieser Senatorengruppe gehören der Partei an, die die Provinzregierung stellt, der dritte kommt aus der Provinzopposition.

\footnotetext{
45 Vgl. Rock, a. a. O., bes. S. 265 ff.

46 Vgl. zu den Verfassungsquellen und ihrem Rangverhältnis Evers, a. a. O., S. 74 ff., Dokumente der ,,Argentinischen Revolution“ im Anhang, S. 243 ff.; vgl. außerdem Vanossi, a. a. O.

47 Vgl. Fix-Zamudio, a. a. O.
} 
Verfassungsrechtlich ist die Bundesregierung, die im Sinne des anglo-amerikanischen ,,government" $z u$ verstehen ist, nach dem Gewaltenteilungsprinzip organisiert, und zwar nicht im Sinne einer strikten Trennung, sondern der funktionellen Zuordnung der Gewalten ${ }^{48}$. Die vollziehende Gewalt übt die oberste Regierungsfunktion aus und steht der allgemeinen Verwaltung der Nation vor (Art. 86 I). Sie hat aber auch gesetzgebende Funktionen, denn sie ist an der Festlegung des Gesetzgebungsinhaltes beteiligt (Art. 86 IV), verkündet die Gesetze, hat ein wichtiges Veto-Recht (Art. 72) und erläßt Durchführungsbestimmungen (Art. 86 II). Lehre, Gesetz und Rechtsprechung haben der Zentralexekutive auch richterliche Funktionen übertragen, die über das Recht, Begnadigungen zu gewähren oder Strafen umzuwandeln (Art. $86 \mathrm{VI})$, hinausgehen.

Die Legislative legt vor allem den Inhalt der Gesetze fest, hat aber auch exekutive Funktionen bei der Ernennung bestimmter Funktionsträger. Das Verfahren der Verfassungsklage gegen bestimmte Hoheitsträger gibt dem Kongreß auch beschränkte richterliche Aufgaben. Die Rechtsprechung, die wie in den USA zwei Arten von Richtern zugewiesen ist, Provinzund Bundesrichtern, entscheidet nicht nur private Streitfälle, sondern kontrolliert auch die beiden anderen Gewalten, indem sie Gesetze und Dekrete für verfassungswidrig erklären kann. Der Oberste Gerichtshof hat die von Marshall in Marbury v. Madison entwickelten Grundsätze des US Supreme Court übernommen ${ }^{49}$.

Die wichtigste verfassungsrechtliche und politische Position nimmt jedoch der Staatspräsident ein, der nach Art. 76 u. a. der römisch-katholischen Kirche angehören muß, die besondere verfassungsrechtliche Protektion genießt und zu der sich die überwältigende Mehrheit der Bevölkerung auch heute noch zumindest der Form nach bekennt. Der Präsident wird nicht vom Kongreß, sondern indirekt durch Wahlmänner gewählt. Die Wahlperiode betrug früher sechs Jahre (Art. 77 und 88), gegenwärtig beträgt sie nach der letzten vorläufigen Regelung vier Jahre mit der, von der früheren Verfassungsrechtslage abweichenden Möglichkeit der unmittelbar anschließenden, einmaligen Wiederwahl. Die der USA-Verfassung nachgebildete Institution des Vizepräsidenten ist bedeutungslos, solange der Präsident das Amt ausübt. Schon verfassungsrechtlich zeigt sich insbesondere bei einem Blick auf die Aufzählung der Befugnisse des Staatspräsidenten in Art. 86 ein eindeutiges Ubergewicht der Spitze der nationalen Exekutive über die anderen Bundesorgane. Darüber hinaus hat die historische Entwicklung des Präsidialprinzips in Argentinien bewiesen, daß der Präsident dauernd mehr Rechte und Befugnisse in Anspruch nimmt, als in der formellen Verfassung vorgesehen ${ }^{50}$. Die Ausdehnung der Macht des Präsidenten in der argentinischen Verfassungsgeschichte ist zugleich die Kehrseite des Abstiegs der Provinzautonomie. Die verfassungsrechtliche Ausgestaltung des Föderalismus hat dieser Zentralisierungstendenz kaum wirksame Grenzen setzen können:

In Art. 1 stellt die argentinische Verfassung fest: ,,La Nación Argentina adopta para su gobierno la forma representativa republicana federal, según la establece la presente Constitución." Bereits in der Präambel wird auf die Bedeutung der Provinzen aufmerksam gemacht. Die Nation setze sich aus den Provinzen zusammen, durch deren Willen und Wahlen sich die Verteter des Volkes in der verfassunggebenden Versammlung zusammengefunden haben. Das bundesstaatlichePrinzip des Staatsaufbaus führt im zweiten Teil der Verfassung zu einer systematischen Gliederung der ,,Autoridades de la Nación“ in ,,Gobierno Federal“(Titel I, Art. 36-103) und „Gobiernos de Provincia“ (Titel II, Art. 104-110).

48 Siehe Ramella, a.a. O., S. $609 \mathrm{ff}$

49 Für die Belege der Rechtsprechung des Obersten Gerichtshofes wird auf den Aufsatz von Ramella verwiesen.

50 Ramella, a. a. O., S. 610, mit einem Hinweis darauf, daß es nicht genüge, den Text der verfassungsrechtlichen Bestimmungenzu lesen, weil die geschriebene Verfassung ständig durch die Praxis und durch neue Bräuche neu ausgelegt und revidiert werde. 
Der Ideengeschichte des „,nacionalismo, centralismo und federalismo“ vor der Verfassung von 1853 kann hier nicht nachgegangen werden ${ }^{51}$. Die Entstehungsgeschichte des Verfassungstextes belegt jedoch eine ausdrückliche Bezugnahme auf das Modell der Bundesverfassung der USA bei der Beratung des Art. ${ }^{52}$. Später hat auch der Oberste Gerichtshof entschieden, daß der argentinische Föderalismus dem der USA außer in den Fällen gleich sei, die juristisch anders lägen ${ }^{53}$. Die Kompetenz, über diese Unterschiede zu befinden, wurde den argentinischen Gerichten, die sich oft an der Rechtsprechung nordamerikanischer Gerichte bei der Interpretation des argentinischen Rechts orientierten, vorbehalten. Bereits 1865 entschied der Oberste Gerichtshof, daß die Bundesjustiz, wie in den USA von der Provinzgerichtsbarkeit unterschieden, nicht der Tradition der spanischen Gesetzgebung folge, da der Verfassungsgeber in dieser Hinsicht die Verfassung der USA nachgeahmt habe ${ }^{\mathbf{5 4}}$. Jedoch gibt es rechtliche Unterschiede zu dem Vorbild. So weist die argentinische Verfassung der Gesetzgebung des Bundes die Kompetenzen im Bereich des Zivilrechts, des Strafrechts, des Handels- und Bergbaurechts, des Arbeitsrechts und des Sozialversicherungsrechts zu (Art. 67 XI). Ferner stellt die Zuweisung der Kompetenzen in den Bereichen Staatsbürgerschaft, Konkurse, Geldfälschung und Laienrichterverfahren an den Kongreß einen klaren Rezeptionsirrtum dar ${ }^{55}$. Diese Befugnisse sind in den USA dem Bund ausnahmsweise gegeben. Die besondere Betonung des Föderalprinzips in der argentinischen Verfassung entsprach den Interessen der Provinzen, gegen unitarische Bestrebungen unter der direkten Herrschaft von Buenos Aires die Hafenstadt lieber mit der Küstenregion bundesstaatlich einzubinden und deren Vormachtstellung politisch zu begrenzen. Die spätere Verfassungsgeschichte machte deutlich, daß die verfassungsrechtlichen Mittel dazu nicht in der Lage waren. Freilich hieß die historische Frage der nationalen Einheit Argentiniens im 19. Jahrhundert nicht: Zusammenschluß - ja oder nein, sondern vielmehr nur: unter welchen Bedingungen, in welcher Form und in wessen Interesse. Die wirtschaftliche Angewiesenheit von Hinterland und Buenos Aires war wechselseitig und verlangte objektiv die politische Einheit, die zur Erfüllung der Aufgaben im Interesse aller Provinzen erforderlich war. Im Kern schien die Interessenlage ein ähnliches Grundproblem aufzuwerfen, wie es Tocqueville in seiner glänzenden Analyse der amerikanischen Bundesverfassung formulierte: ,,Es handelte sich darum, die oberste Gewalt so aufzuteilen, daß die Staaten, welche die Union bildeten, sich weiterhin in allem selbst regierten, das nur ihre innere Wohlfahrt betraf, ohne daß die in der Union verkörperte Gesamtnation aufhörte, eine Körperschaft zu sein und für alle die Gesamtheit betreffenden Bedürfnisse aufzukommen. Eine verwickelte und schwer zu lösende Frage“"56. Bei der Frage der ausgewogenen Teilung der Souveränität ${ }^{57}$ im Sinne der Rechtssetzungsbefugnis und Machtausübung ging es den nordamerikanischen Einzelstaaten darum, der Bundesgewalt sowenig Kompetenzen wie möglich und soviel wie zur Erledigung der gemeinsamen Aufgaben erforderlich zu übertragen. Deshalb beließ die Verfassungskonstruktion den Gliedstaaten das gemeine Recht zur eigenen Regelung und errichtete die Befugnisse der Bundesgewalt im Grundsatz als Ausnahme. Ihre Kompetenzen wurden sorgfältig aufgezählt, alles andere sollte den Einzelstaaten vorbehalten bleiben.

\footnotetext{
51 Vgl. dazu J. L. Romero, Las ideas políticas en Argentina, 3. Aufl., México-Buenos Aires 1959.

52 Nachweise: Corpus Constitutionnel, a. a. O., S. 355.

$53 \mathrm{Vgl}$. Ramella, a. a. O., S. 598.

54 a. a. O., S. 622 .

55 a. a. O., S. 620

56 Alexis de Tocqueville, Úber die Demokratie in Amerika, hrg. v. Mayer/Eschenburg, Zbinden, München 1976, S. 128 f.

57 Zum schillernden Souveränitätsbegriff vgl. J. Dennert, Ursprung und Begriff der Souveränität, 1964; Tocqueville, a. a. O., S. 187, versteht ihn als Hoheitsgewalt vor allem im Hinblick auf die Rechtssetzungsbefugnis und Machtausübung.
} 
Die Begründung des Entwurfs der argentinischen Bundesverfassung durch den Berichterstatter des Verfassungsausschusses zeigt den gleichen Denkansatz ${ }^{58}$. Theoretisch erschienen auch hier die Provinzen als das Originäre und Souveräne, das die eigene Existenzweise prinzipiell selbst bestimme, während die Zentralregierung für bestimmte Aufgaben als Ausnahme eingerichtet wird. Nach der Grundregel des argentinischen Föderalismus in Art. 104 haben die Provinzen alle Gewalt, soweit diese nicht durch die Verfassung der Bundesgewalt eingeräumt ist, sowie die Rechte, die ihnen durch Sonderverträge bei ihrer Eingliederung in den Föderalverband vorbehalten wurden. Der Zusatz wurde durch die Verfassungsreform von 1860 eingefügt. Das Verteilungsprinzip des Art. 104 geht also davon aus, daß die Gewalt der Provinzen prinzipiell unbegrenzt, die der Bundesgewalt grundsätzlich begrenzt sei. Die konkrete Verteilung der Kompetenzen selbst allerdings stellt dieses Prinzip auf den Kopf. Der Zentralgewalt wurden weit mehr, zudem der ausdehnenden Auslegung fähigen Befugnisse eingeräumt, als dies bei dem Vorbild der USA-Verfassung geschehen war.

Wichtige Fundamentalnormen, die die Autonomie der Provinzen garantieren, finden sich im ersten Teil der Verfassung. Die Provinzen stellen der Grundidee nach keine bloßen Verwaltungseinheiten, sondern politisch autonome Gebietskörperschaften dar. Art. 13 enthält hinsichtlich des Bestandsschutzes seinem Wortlaut nach eine flexible Regelung, die unter bestimmten Bedingungen Gebietsveränderungen zuläßt. In einer Entscheidung aus dem Jahre 1937 hat der Oberste Gerichtshof aus Art. 13 abgeleitet, daß die Provinzen unzerstörbar seien ${ }^{59}$. Dabei handelt es sich nur um eine institutionelle Garantie und nicht um die Verewigung der konkreten Gestalt der bestehenden Provinzen.

Art. 5 stellt die Bedingungen auf, die ein Mindestmaß an Ubereinstimmung zwischen der Bundesverfassung und den Verfassungen der Provinzen gewährleisten sollen. Danach gibt sich jede Provinz eine Verfassung im Rahmen des repräsentativen republikanischen Systems gemäß den Grundsätzen, Erläuterungen und Garantien der nationalen Verfassung. Insbesondere habe die Provinzverfassung die Rechtspflege, die kommunale Selbstverwaltung und die Grundschulerziehung zu gewährleisten. Unter diesen Voraussetzungen wird der Bundesgewalt die Pflicht auferlegt, jeder Provinz den Genuß und aie Ausübung ihrer Funktionen zu sichern. Einschlägig in diesem Zusammenhang sind außerdem Art. 105 und 106. Gemäß Art. 105 soll jede Provinz die eigenen Institutionen einrichten und von diesen regiert werden. Sie sollen ferner, ohne Einmischung durch die Bundesgewalt, die eigenen Gouverneure, Gesetzgeber und Funktionärsträger wählen. Der Substanz nach bestätigt ${ }_{1}$ Art. 106 nur noch einmal Art. 5 und hält fest, daß jede Provinz die eigene Verfassung erläßt, wie in Art. 5 vorgesehen.

Die Form der Ausübung der Autonomie der Provinzen ist nicht gerichtlich überprüfbar. Denn der Oberste Gerichtshof hat es prinzipiell abgelehnt, sich in diese Frage einzumischen $^{60}$.

Aus Art. 105 folgt, daß die Gesetzgebung der Provinzen das Provinzwahlrecht bestimmt. Diese Norm ist eindeutig verletzt worden, als die „,De-facto-Regierungen“ von 1955 und 1962 den Provinzen Wahlsysteme aufzwangen, die von den Verfassungen und einfachen Wahlgesetzen der Provinzen völlig verschieden waren ${ }^{\mathbf{6 1}}$.

Nach Art. 110 sind die Provinzgouverneure ,, agentes naturales del Gobierno federal“, um die Bundesverfassung und die nationalen Gesetze auszuführen. Die Gesetzgebungskompetenz und die Finanzquellen der Bundesgewalt sind der Sache nach, insbesondere durch eine

58 Vgl. Corpus Constitutionnel a. a. O., S. 355.

59 Ramella, a. a. O., S. 598.

60 a. a. O.

61 a. a. O., S. 629. 
Praxis der ausdehnenden Auslegung, in entscheidenden Bereichen kaum begrenzt, während den Provinzen materiell nur Bereiche von sekundärer und tertiärer Bedeutung überlassen sind (Art. 107, 108, Art. 67 und 86) ${ }^{62}$. Besonders gefährlich für die Autonomie der Provinzen hat sich die Befugnis der Zentralgewalt erwiesen, über die Gewährung von nationalen Mitteln an finanzschwache Provinzen zu entscheiden. Das Recht der Provinzen nach Art. 107, ihre Industrie, die Einwanderung, den Bau von Eisenbahnlinien und Schiffahrtsstraßen, die Besiedlung des eigenen Landes, die Einführung und Errichtung neuer Industrien, die Kapitaleinfuhr und die Flußnutzung mit Schutzgesetzen und eigenen Mitteln zu fördern, sind ohne Zuschüsse aus Buenos Aires weitgehend nur eine verbriefte Hoffnung. Dieses finanzielle Druckmittel in den Händen der Zentralregierung ist ein Zentralisierungsmittel erster Ordnung. Rechtlich werden die Provinzkompetenzen dadurch eingeschränkt, daß es zur Praxis des Kongresses gehört, auf Gebieten, auf denen es an einer Bundeskompetenz ausdrücklich fehlt und die auch nicht über die Lehre von den „Podres Implícites“ begründet werden kann, sog. ,,Gesetzesempfehlungen“ zu verabschieden, die dann von den Provinzen in eigener Kompetenz übernommen werden. Diese Verfassungsgewohnheit ist nicht zuletzt auf die Finanzabhängigkeit der Provinzen zurückzuführen.

Das entscheidende verfassungsrechtliche Mittel, das zur Aushöhlung der Provinzselbständigkeit von Anfang an geführt hat, ist allerdings der Mißbrauch des Interventionsrechts in Art. 6, das sich als gefährliche politische Waffe in den Händen der verschiedensten Regierungen dargestellt hat. Dieses Recht der Zentralgewalt existiert auch in anderen Bundesverfassungen, etwa in der Schweiz oder den USA, hat aber dort niemals eine ähnliche oder vergleichbare Funktion wie in Argentinien eingenommen. Das Interventionsrecht unterscheidet vier Fälle. Die Bundesgewalt kann die Initiative ergreifen, um entweder die demokratisch-republikanische Regierungsform zu sichern oder um eine fremde Invasion abzuwehren. Weniger relevant sind die beiden anderen Fälle, die von einem Ersuchen der Provinzorgane ausgehen, wenn sie durch Hochverrat abgesetzt worden sind oder der Invasion durch eine andere Provinz ausgesetzt sind.

Zwar steht nach der Rechtsprechung das Interventionsrecht im ersten Fall grundsätzlich dem Kongreß $\mathrm{zu}^{63}$, in dem die Provinzen zumindest rechtlich im Senat ein Mitspracherecht haben. In der Praxis aber wurde es meist von der Exekutive, d. h. von dem jeweiligen Inhaber des Präsidentenamtes, sei er Zivilist, sei er Militärangehöriger, in Anspruch genommen. Juristische Möglichkeiten, dem Mißbrauch des Interventionsrechtes einen Riegel vorzuschieben, bestehen nicht. Der Oberste Gerichtshof will unter Beachtung des ,,judicial selfrestraint" und der ,,political question“ Doktrin nicht in ausgesprochen politische Verfahren eingreifen, wozu die Anwendung der Intervention gerechnet wird. Somit gehört die Feststellung der Voraussetzungen der Intervention zu den unnachprüfbaren Privilegien der Bundesgewalt unter dem Regiment des Präsidenten der Republik. Die Rechtsprechung beschränkte sich darauf, juristische Kategorienfür das, was geschah, zu entwickeln. Der staatliche Inventor sei ein Sondervertreter des Präsidenten. Obwohl er nationaler Beamter sei, wird fingiert, daß seine Tätigkeit die rechtliche Wirkung habe, als werde sie von den Provinzautoritäten selbst vorgenommen.

In der Zeit von 1854 bis 1940 sind in Argentinien etwa 140 Interventionen der Zentralmacht zu verzeichnen, wobei vermutet wird, daß die Frequenz danach eher zugenommen hat. Dabei ist es interessant, daß allein in dem Zeitraum von 1860 bis 1930, also bis zum ersten Militärputsch, mehr als 100 dieser Akte registriert wurden ${ }^{64}$. Tatsächlich hat die Verfassungs-

$62 \mathrm{Vgl}$. die Analyse in Corpus Constitutionnel, a. a. O., S. $358 \mathrm{f}$.

63 Ramella, a. a. O., S. 600.

64 Vgl. Corpus Constitutionnel, a. a. O., S. 359. 
praxis die Intervention per Dekret des Präsidenten sanktioniert. Diese Praxis etablierte sich besonders unter Yrigoyen zwischen 1916 und 1922, der etwa 20 Male in den Provinzen Gouverneure, Gesetzgeber und Funktionsträger absetzen und ersetzen ließ ${ }^{65}$. Von den Militärregierungen wurde diese Praxis nur übernommen und nicht eingeführt. Die Prozedur ist als Zentralisierungs- und politisches Disziplinierungsmittel ebenso einfach wie wirkungsvoll. Ein Dekret des Präsidenten setzt einen Bundeskommissar ein. Dieser übernimmt zeitweilig die Funktion des Gouverneurs an der Spitze der Provinzgewalt mit umfassenden Vollmachten. Funktionäre und Politiker werden solange ersetzt und die Provinzgesetzgebung suspendiert, bis die politischen Ziele der Zentralgewalt erreicht sind und die in Buenos Aires herrschende Partei sich auch in der Provinz durchgesetzt hat.

$\mathrm{Zu}$ den institutionellen Maßnahmen der Militärputsche 1930, 1943, 1955, 1962, 1966 und 1976 gehörten nicht immer gleichzeitig die Auflösung aller politischen Parteien bzw. das Verbot der politischen Betätigung, die Verhängung des Belagerungszustandes, die Umbesetzung des Obersten Gerichtshofes oder der Erlaß eines geschriebenen Revolutionsstatutes. Zu den regelmäßigen Maßnahmen ohne Ausnahme gehörten jedoch die Absetzung des Präsidenten und die Aufhebung der Provinzautonomie ${ }^{66}$.

Die Zentralisierungstendenz ist durch die Herrschaft der Militärregime beschleunigt worden. Die kommissarischen Eingriffe in die Provinzautonomie durch das Militär hatten in den Fällen, in denen die formale Struktur der Provinzverfassungen wiederhergestellt wurde, mehr psychologische denn machtpolitische Bedeutung ${ }^{67}$. Für den Niedergang des bundesstaatlichen Prinzips sind jedoch die vorangegangenen Zivilregierungen und die allgemeine sozio-ökonomische Entwicklung Argentiniens nicht weniger verantwortlich wie die Militärdiktaturen ab 1930.

Probleme eines ,,ethnischen Föderalismus“ hat Argentinien mit seiner relativ homogenen kulturellen und ethnischen Zusammensetzung nie gekannt. Die Probleme des argentinischen Föderalismus waren im 19. Jahrhundert zunächst machtpolitischer Natur im Prozeß der nationalen Einigung und haben in diesem Jahrhundert zunehmend den Charakter des Gegensatzes zwischen Buenos Aires und dem Hinterland in einem ökonomischen und infrastrukturellen Sinne und zwischen reich und arm in einem sozialen Sinne angenommen. Diese Probleme können nicht isoliert von den komplexen Erscheinungen der Dauerkrise Argentiniens gesehen und gelöst werden. Im heutigen Argentinien in seiner unitarischen Gestalt der politischen Realverfassung und mit der Kraftlosigkeit des formellen und materiellen Verfassungsrechtes stehen weder Fragen des ,,kooperativen“ Bundesstaates noch die Rückkehr zu den föderalistischen Prinzipien der Verfassung von 1853 auf der Tagesordnung. Das nordamerikanische Verständnis, daß sich Föderalismus und Demokratie wechselseitig bedingen und ergänzen, daß Demokratie im Grunde nur als föderative denkbar sei und daß schließlich letztere vor allem auf ein System des , ,limited government“" und der gegenseitigen ,,schecks and balances" in der Zuordnung der Staatsorgane zur Abwehr von Machtkonzentration und Machtmißbrauch beruhe, hat in Argentinien keinen nährreichen Boden finden können. Aber nicht nur die geistige, politische und rechtliche Kultur und Tradition, sondern auch die geographischen, wirtschaftlichen und sozialen Bedingungen waren in Argentinien 1853 und später bei der Rezeption der nordamerikanischen Bundesverfassung zu verschieden von den Voraussetzungen des etwa 70 Jahre älteren Vorbildes. Zu der mexikanischen Verfassung

651922 waren Bundesinterventionen in die Provinzen durch einfaches Verwaltungsdekret ohne Einschaltung des Kongresses und erhöhte Staatsausgaben durch Dekret oder Ministerbeschlüsse zu den Hauptpfeilern des patronage-Systems geworden, auf dessen Grundlage Yrigoyens System der Kontrolle durch die Partei arbeitete, vgl. Rock, a. a. O., S. 222.

66 Eine Ubersicht über die institutionellen Maßnahmen von Revolutionsregierungen anläßlich des Umsturzes bis zum Putsch 1966 gibt Evers, a. a. O., S. 52.

67 Für die "Argentinische Revolution" und danach vgl. Evers, a. a. O., S. 90 ff. 
von 1824 schrieb Tocqueville: ,Die Verfassung der Vereinigten Staaten gleicht jenen schönen Schöpfungen menschlicher Arbeit, die ihre Erfinder mit Reichtum und Gütern überschütten, die aber in anderen Händen unfruchtbar bleiben. Das hat in unseren Tagen Mexiko gezeigt. Die Bewohner Mexikos, welche die Bundesordnung einführen wollten, nahmen die Bundesverfassung ihrer Nachbarn, der Angloamerikaner, zum Vorbild und ahmten sie fast vollständig nach. "Unter Hinweis auf den damaligen mexikanischen Zyklus zwischen Anarchie und Militärdespotie fährt der französische Aristokrat in seinem Plädoyer für die Gleichheit fort: ,,Vermochten Sie den Buchstaben des Gesetzes zu übernehmen, so konnten sie sich nicht gleichzeitig den Geist, der es beseelt, aneignen." 68 Wenn man jedoch der Ansicht ist, daß eine „Verfassung“ mehr als nur ein Produkt des Geistes, ein ,,Gedankending“ ist - eine Sicht, die Hegel in den ,Grundlinien der Philosophie des Rechts“69 skeptisch gegenüber dem Versuch stimmt, ,,einem Volke, eine wenn auch ihrem Inhalt nach mehr oder weniger vernünftige Verfassung a priori geben zu wollen“-, so wird man im Hinblick auf Argentinien bei dem Scheitern der Verfassungsrezeption nicht nur die verschiedene Lebensart und politische Kultur, sondern vor allem auch die extern und intern anders bedingten materiellen Voraussetzungen und sozio-ökonomischen Strukturen hervorheben müssen. Das „Vernünftige“ kann dort nicht wirklich werden, wo die vernünftigen Ideen aus einer anderen Wirklichkeit, aus anderen historischen Bedingungen entspringen.

68 Tocqueville, a. a. O., S. 187.

$69 \mathrm{Vgl}$. insbesondere $\ 274$ Rechtsphilosophie. 
These criticisms should, however, serve to refine the theory and develop it further. The Egyptian case study and the empirical testing it involved revealed Huntington's framework to be a useful tool in empirical research but one needing to be developed considerably and used in conjunction with insights from other disciplines.

\title{
Customary Law in Togo under German Colonial Rule
}

\author{
By E.A.B. van Rouveroy van Nieuwaal
}

Many books and articles have been written about the legal policy of the colonial powers in their former colonies. At the beginning of this century the formal legal relationship between the imported law (from European or Islamic origin) versus customary law drew the attention of the colonial lawgiver and scholars as well. About German legal policy in the colonies little is known outside the German (scientific) world. One of the reasons may be the comparatively short time that Germany was a colonial power; their colonial empire lasted only from $1884 / 1885$ till 1919, the year of the Treaty of Versailles.

In this contribution the author describes in detail the legal framework of German law and "Gewohnheitsrecht" ("indigenous law") in one of Germany's best known colonies, Togo, in West Africa. Particularly the position of Togolese land tenure law comes up for discussion.

\section{Federalism under Military Government: Argentina}

By Peter Malanczuk

The decline of federalism and of provincial autonomy guaranteed by the Argentine Constitution of 1853 with its later amendments is a significant examplefor the problems concerning the effectiveness of Argentine constitutional law in general. The "Provincial Problem" has marked Argentine history under changing conditions from the declaration of independence to the present days of military rule. The degrading of the provinces to administrative branches of civil or military central governments in the dominant capital region of Buenos Aires is due to complex economical, technical, political and constitutional factors. This essay is primarily concerned with the constitutional aspects which, however, cannot be separated from the institutional crisis and the underlying structural problems of socio-economic development and change since the first successful military coup in 1930. The present political situation in Argentina, since 1976 again as in most of the time after 1930 under military rule, has to be seen in this context (I.). To understand the process of the decline of federalism which started before the formal abolition of provincial autonomy became one of the standard measures of the various military regimes, it is necessary to consider the historical background in the 19th century and the development of the "Provincial Problem" (II.). The constitutional arrangement of federalism, the constitutional practice and the gap between constitutional text and reality are examined on this basis (III.). The development of Argentina to a unitary state in substance in contrast to the Federal Constitution of the USA which served as a model is to be explained by the entirely different geographical, economical, political and cultural-legal conditions. Military rule has enforced the tendency towards centralisation but previous civil governments are no less responsible for the decline of federalism. 